\title{
Preparing parents for parenthood: protocol for a randomized controlled trial of a preventative parenting intervention for expectant parents
}

\author{
Mandy Mihelic ${ }^{1 *}\left(\mathbb{D}\right.$, Alina Morawska ${ }^{1}$ and Ania Filus ${ }^{2}$
}

\begin{abstract}
Background: Becoming the parent of a new baby comes with a range of challenges including difficulties with emotional adjustment, couple relationship issues and difficulty managing common infant behaviors, such as crying and sleep problems. This time can be especially challenging for couples who experience a range of risk factors. Previous parenting interventions for parents of babies have shown mixed results. This protocol paper describes a randomized controlled trial of a group-based parenting intervention for high-risk parents expecting their first baby.

Methods/design: Participants will be randomized to either Group Baby Triple P or Care as Usual (CAU). Group Baby Triple P involves $4 \times 2 \mathrm{~h}$ group sessions delivered during pregnancy and 4 individual telephone sessions of $30 \mathrm{~min}$ each in the early postnatal period. Outcomes will be assessed via parent self-report questionnaire, home observations and a baby diary 10 weeks and 6 months post-birth. Primary outcomes will be parental confidence and perceived competence. Secondary outcomes will include parental responsiveness and bonding with the baby, relationship happiness, life satisfaction, depression, anxiety and stress, and infant crying and sleep. Analyses will involve a series of rANOVA and rMANOVAs, t-tests and a multilevel modeling approach.
\end{abstract}

Discussion: A brief summary, strengths and potential implications are discussed.

Trial registration: Australian New Zealand Clinical Trials Registry: ANZCTR 126130009487796. Registered 27 August, 2013.

Keywords: Parenting intervention, Preventative parenting, Infants, Randomised controlled trial

\section{Background}

The transition to parenthood comes with a range of challenges. New parents are faced with sleep deprivation, mastering infant caregiving tasks, changes in their relationship and substantial lifestyle changes [1,2]. Postnatal depression in both mothers and fathers is relatively common and its presence has many short- and longterm adverse effects for children's cognitive, social and emotional development $[3,4]$. A significant proportion of couples feel less satisfied with their relationship after becoming parents and couple conflict often increases [5]. At the same time, these early months of family formation are crucially important for the infant's mental health and development as well as for the mental wellbeing of parents. It is widely accepted that dysfunctional parenting practices and family conflict are generic risk factors that impact on a child's development and contribute to the occurrence of emotional and behavioral problems from a very young age [6]. Longitudinal studies have shown that stress and distress in parents during the early years of family formation lead to negative outcomes for children lasting into the preschool and primary school years $[7,8]$. Therefore, preparing couples for the transition to parenthood and supporting new parents in their new role is a worthwhile investment.

\footnotetext{
*Correspondence: mandy.mihelic@uq.net.au

${ }^{1}$ Parenting and Family Support Centre, School of Psychology, The University

of Queensland Brisbane, St Lucia, QLD 4067, Australia

Full list of author information is available at the end of the article
}

\section{Difficulties experienced at the transition to parenthood}

Becoming a parent means adjusting to many changes in life and this process of psychological adaptation can 
place a strain on one's mental health as well as on one's personal relationships. It also means managing common, albeit distressing baby behaviors, such as problems with sleeping and crying. Severe sleeping and crying problems affect approximately $20 \%$ of infants $[9,10]$. Before the birth of their first baby, less than half (44\%) of parents report feeling adequately prepared for parenthood. After their child is born, a mere $18 \%$ report feeling confident as parents [11]. As many as a third of women report problems caring for themselves and their baby and many women, especially first time mothers view current postnatal services as inadequate [12]. In addition to this lack of confidence, some mothers and fathers experience more severe, negative adjustment difficulties, such as symptoms of depression [up to 22\%],[13], lowered self-esteem, anxiety [up to 15\%]; [14], feelings of fatigue and extreme exhaustion [15-20].

The couple relationship is equally challenged during this period: numerous studies have shown a decrease in relationship satisfaction alongside a decrease in quality time spent together, positive communication and sexual activity $[15,21]$. Low relationship satisfaction is associated with increased conflict, individual psychological distress, negative parentchild relationships and negative child outcomes $[22,23]$.

\section{Risk and protective factors at the transition to parenthood}

Several identified risk factors at the transition to parenthood are associated with a range of negative outcomes for parents, and subsequently their children. Non-modifiable risk factors include low socio-economic status and unplanned pregnancy [24-27]. A range of modifiable risk factors include prenatal depression and anxiety [28], low relationship satisfaction [22, 23], low social support $[29,30]$ and low confidence [31].

In particular, anxiety and depression in the prenatal and postnatal period have been associated with adverse outcomes for the child, including child maladjustment and internalising difficulties [28], negative behavioural activity at four months of age [32] and lower infant cognitive and psychomotor development [4]. Therefore, addressing these risk factors early on is crucial in preventing poor child development and behavioural outcomes.

The impact of a negative couple relationship can also lead to detrimental child outcomes. Research suggests that problems in the couple relationship can spill over onto the parent-child relationship [33], which in turn can negatively and indirectly contribute to the child's development through changes in parenting [34]. For example, couple conflict can influence the child's mental health through an effect on the child's emotional security [35].

The parenting aspect itself is also an important contributor to child outcomes. Parental self-efficacy and maternal/ paternal responsiveness are two protective factors that have been linked to better outcomes for parents of infants. Parental self-efficacy or confidence is related to more realistic parenting expectations during pregnancy [36], better coping with challenges of the early postnatal period, better couple relationship quality, greater satisfaction with their infants [37], reduced depression [38] and more competent and positive parenting skills $[39,40]$. Self-efficacy has also been related to the second protective factor, responsiveness, which refers to positive parental interactive behaviors and sensitivity to infants [41]. Sensitive responsiveness aids in the parents' ability to understand and respond to infant signals [39] and is an important factor in the development of secure infant attachment [42]. A link to improved infant sleep has also been suggested [43] as well as in reducing infant crying problems [44].

\section{Previous interventions at the transition to parenthood}

The etiology of emotional and behavioral problems in early childhood is complex, and both genetic and environmental factors play a role. However, of all the potentially modifiable risk and protective factors that can affect a child's development, improving parenting skills and confidence holds the greatest potential in improving health and wellbeing of children as well as the family as a whole [6]. Although we know that the early family context is crucial, parents generally receive little preparation beyond the experience of having been parented themselves. A metaanalytic review of the effects of parenting education with expectant and new parents [45] found that, on average, interventions had small to very small effect sizes on parenting, parental stress, child development and mental health, parental mental health and couple adjustment. Those interventions which were shorter (3-6 months) in duration, which had a postnatal component and which targeted highrisk parents tended to have better outcomes. A likely reason for the small effect sizes were that prevention programs such as parenting programs during pregnancy and the early postnatal period, tend to have smaller effects as some parents are expected to adjust well regardless of attending a program. Other systematic reviews have reported on positive intervention effects regarding enhanced sensitivity and infant attachment security, responsiveness, and increased infant sleep and maternal knowledge; however there is little data on the effects on self-efficacy and competence [46-48].

Other programs at the transition to parenthood, such as the Family Foundations program and Couple CARE for Parents focus on the coparenting relationship [49-51]. Positive results of the Family Foundations program have been found on coparenting outcomes, infant soothability and self-soothing, mental health among mothers, and fewer parent-child dysfunctional interactions [50]. Improvements after the Couple Care for Parents program 
have been found for the couple relationship and parenting stress levels [52], but no significant results were found in regards to parenting itself. However, both the Couple Care for Parents and the Family Foundations mainly focus on the couple relationship, while the parenting component is minimal.

\section{The triple $\mathbf{P}$ - positive parenting program: Baby triple $\mathbf{P}$}

The majority of existing programs at the transition to parenthood focus on one area of adjustment only (parenting aspects, couple adjustment or individual adjustment) even though research on the transition to parenthood has shown that all three aspects are important when it comes to creating positive outcomes for infants [3, 22, 23, 53]. Baby Triple $\mathrm{P}$ [54] is a psychological parenting intervention aimed to prepare new parents for a positive transition to parenthood by teaching them skills in the domains of parenting their baby, looking after their own wellbeing, as well as maintaining a positive relationship with their partner. Baby Triple P's unique aspect is its self-regulatory framework and active skills training to enhance self-efficacy and encourage parents to generalize their learnt skills to when their child is older or to other areas and times of their lives. It is part of the Triple P - Positive Parenting Program system of interventions [6].

An initial feasibility trial conducted by Spry [55] investigated Baby Triple P in a universal population. Using a sample of 129 couples, results showed that while the couples who participated in the intervention were satisfied with the help they received, none of the outcomes measured showed significant differences between the intervention and the care as usual group. The author noted that while she aimed for a representative sample of Australian first-time couples, the sample they obtained was older, more educated and better off financially, as well as better adjusted than the general population, thus ceiling effects were found on all outcome measures at baseline.

Further studies reported high acceptability and feasibility of Baby Triple $\mathrm{P}$ among families with premature babies [56], mothers in a psychiatric unit [57], and mothers suffering from postnatal depression [58]. However, Tsivos, Calam [58] did not find any significant effects of the intervention for the mothers in the Baby Triple P group compared to the control group, possibly due to the low sample size $(n=$ 27) of this pilot study. Additionally, mothers with clinical depression may need greater psychological support than offered by Baby Triple P.

Other trials include an investigation of Baby Triple $\mathrm{P}$ postnatally on mother-infant attachment and bonding [59]. While parents rated the program as highly acceptable and satisfactory, there was no effect of Baby Triple P on parent responsiveness and other outcome measures, such as postnatal depression in mothers or fathers. Reasons for the non-significant results similarly include low power in detecting an effect $(n=71)$ as well as potential ceiling effects given the sample was relatively advantaged in terms of their sociodemographic status.

Even though previous trials on evaluating the efficacy of the Baby Triple P program have not found significant outcomes of the intervention, plausible reasons for the lack of significant findings, including small sample sizes and ceiling effects, call for an additional randomized controlled trial to investigate the effects further. This is especially due to the high feasibility and acceptability reported by parents in previous studies as well as the need for a program that prepares new parents for their parenting journey. Fortunately, the ceiling effects in previous trials might indicate that despite the challenges of transitioning to parenthood, the majority of new parents seem to adjust quite well. However, the transition to parenthood can be particularly challenging for families who are at greater disadvantage than others and already experience a range of risk factors. Consequently, developing and evaluating antenatal education programs for high-risk parents is crucial in order to prevent negative outcomes for parents and infants. Yet, according to Gagnon and Sandall [60], the majority of studies assessing antenatal parenting interventions often include only well-educated mothers in the middle to upper socio-economic strata, who might do well regardless of which, or any preparation classes they attend, consistent with the prior findings relating to Baby Triple P. From a cost-benefit analysis, focusing on a higher risk sample for this current trial of Baby Triple is reasonable.

\section{Aims and hypotheses}

In light of the critical importance of a successful transition to parenthood it is clear that strategies need to be developed to support families and enhance the protective factors for babies to ensure positive developmental outcomes. This is vitally important especially for those families with identified risk factors who may find the transition to parenthood particularly challenging. The proposed research will evaluate the efficacy of a tailored parenting intervention, Baby Triple P that combines parenting information, with the provision of strategies to enhance individual wellbeing and couple adjustment for highrisk families.

The aim of this study is to experimentally test the hypothesis that provision of parenting support at the transition to parenthood through Baby Triple P, will lead to improvements in parental confidence and sense of competence in their abilities as parents. Furthermore, we will test the secondary hypotheses that the program will lead to higher levels of responsiveness and bonding with the baby, higher relationship happiness, higher levels of life satisfaction, fewer instances of depression, anxiety and stress, and infants would show less fussing or crying and fewer sleeping problems. It is hypothesized 
that these positive treatment effects will be maintained at six-month-follow up.

\section{Method \\ Design}

The study is a 2 (Baby Triple $\mathrm{P}$ vs CAU) $\times 3$ (time: pre-test, post-test, 6-month-follow up) design with multi-informant assessment. The study will experimentally test the relative impact of Baby Triple P against care as usual in a randomized controlled trial aimed at improving baby and parenting outcomes. The CONSORT guidelines for randomized controlled trials will be used. This protocol also adheres to the SPIRIT guidelines.

\section{Ethics}

Ethical approval has been obtained from the Queensland Children's Health Services Human Research Ethics Committee (HREC/13/QRBW/177) and the University of Queensland Behavioural and Social Sciences Ethical Review Committee (2013000564). The trial has been registered with the Australian New Zealand Clinical Trials Registry (ACTRN12613000948796).

\section{Participants}

Participants will be approximately 110 couples (110 mothers and 110 fathers) expecting their first child and between 20 and 35 weeks of pregnancy, recruited through primary care settings, maternity hospitals, obstetricians, and through targeted mail-out and media campaigns within the Brisbane and Ipswich area in Queensland, Australia.

\section{Inclusion and exclusion criteria}

Expectant couples have to meet the following inclusion criteria: (a) experiencing a first pregnancy (between 20 and 35 weeks gestation), (b) in a couple relationship, (c) aged at least 18 years, and d) living in the Brisbane area for the duration of the study. Couples will also have to meet at least two of the following risk factors: i) unplanned pregnancy, ii) low education, iii) low income or experiencing financial strain, iv) low relationship satisfaction, v) low social support, vi) history of maternal/ paternal depression or anxiety, vii) current depression or anxiety, viii) low parenting efficacy, ix) low life satisfaction/ happiness, $x$ ) aged between 18 and 21 years.

Parents will be excluded if: a) they have an intellectual disability or severe psychopathology which would impair their understanding of the material presented in the program, b) parents do not read and understand English, c) they have a diagnosed genetic disorder, disability in the baby, or experience major complications during pregnancy d) parents are currently receiving psychological help or counseling. Eligibility to these criteria will be based on mothers' responses only.

\section{Recruitment}

Participants will primarily be recruited through antenatal clinics at the Royal Brisbane Woman's Hospital and the Mater Hospital (Brisbane, Australia). Recruitment brochures and posters will be distributed in antenatal clinics. Potential participants will also be approached during antenatal classes. Registration will be via phone, email or on the study website. Parents will have the opportunity to discuss any questions or concerns regarding participation with one of the research team.

Recruitment brochures and posters will be distributed at GP clinics, obstetrician clinics, baby shops and local libraries across the Greater Brisbane, Ipswich and Logan area. This information will also be available through advertisements posted on baby websites and forums, and social media pages (e.g., Facebook), as well as advertisements in the University of Queensland staff newsletter and articles in local newspapers.

Interested parents will be directed to the study website. Mothers will receive information and consent forms detailing the project, and will have the opportunity to address any concerns or questions with the project coordinator before giving consent, and being directed to the online self-report questionnaire. Eligibility will be based on the mother's responses only and her response will be used to determine if she is eligible based on the inclusion and exclusion criteria a) to d) outlined above. If eligible based on these criteria, the total number of risk factors ( $\mathrm{i}$ to $\mathrm{x}$ ) will be summed and if a minimum of two risk factors are present, the mother will be deemed eligible to take part in the trial. Then the father will be asked to complete the self-report questionnaire as well. Following this, the couple will be enrolled in the study by the study coordinator.

\section{Randomization}

Participant randomization to either the Baby Triple P or CAU group will be conducted by generating a random allocation sequence, by a researcher not involved in the project, using a computer-based random number generator. A pre-prepared series of sealed opaque envelopes, each labelled with a participant ID number, will conceal group allocation from researchers and participants until after completion of the baseline assessment. Immediately after completion of the baseline questionnaires, the research coordinator will open the envelope, and will notify participants of their condition allocation via phone.

Participants allocated to the intervention condition will attend group sessions based on their preference for day and time, depending on availability of groups. Participants will be allocated to groups as soon as possible after randomization. Figure 1 summarizes the flow of participants through the study. 


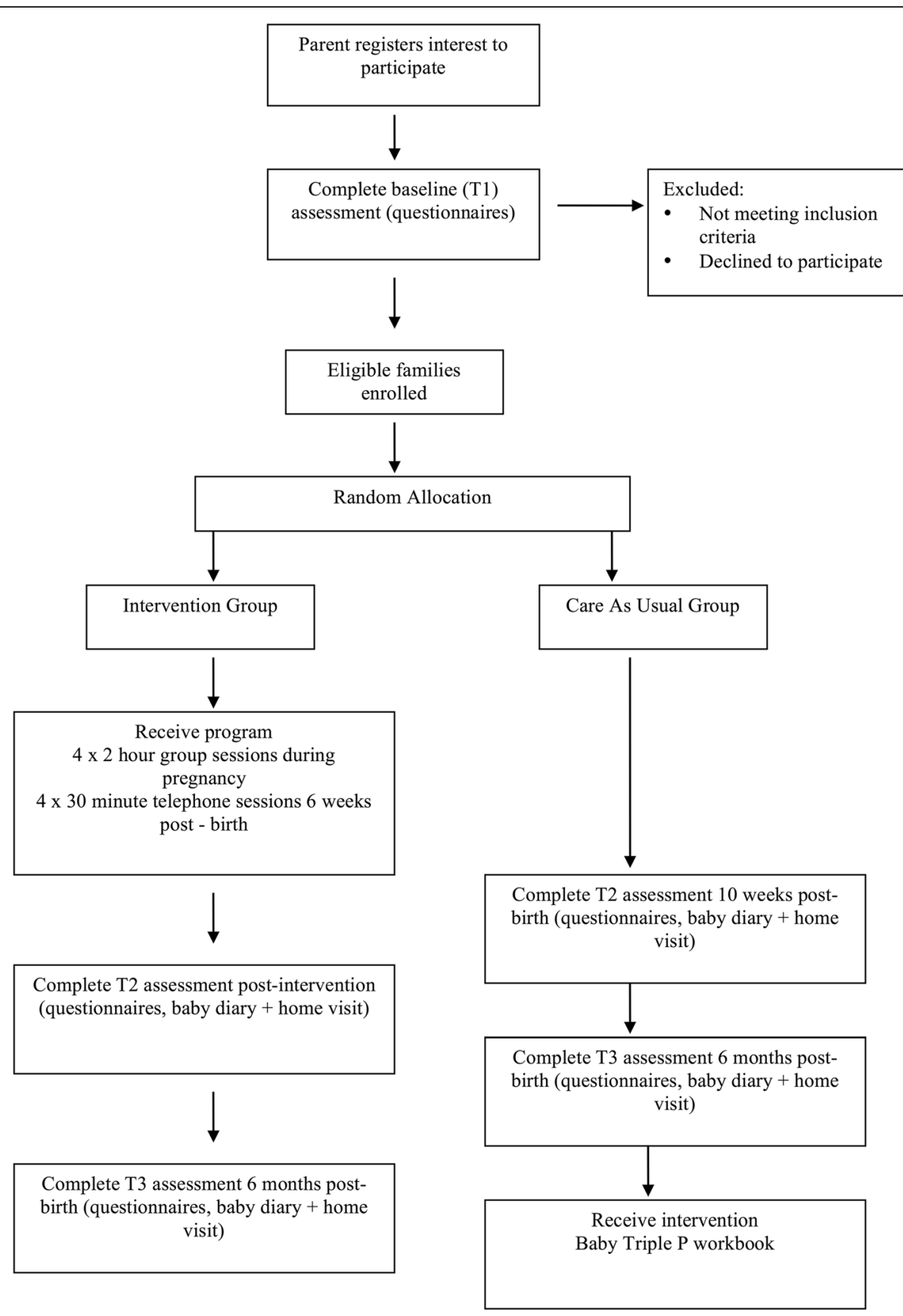

Fig. 1 Participant flow through the study

\section{Intervention}

The intervention will consist of four, 2-h Baby Triple $P$ group sessions conducted during pregnancy, followed by four individual 30-min telephone sessions conducted postnatally. The program is based on the same theoretical principles that underpin the Triple P system [6] and sessions are interactive and allow opportunities for discussion.

Baby Triple P is an eight-session psychological intervention, developed by Spry (2013) that aims to prepare couples for a positive transition to parenthood by teaching them core skills in the domains of parenting their baby, taking care of their own wellbeing and maintaining a positive relationship with their partner. The self-regulatory framework will be used to engage participants and facilitate learning. Specifically, it includes active training methods, for example modelling, rehearsal, practice, feedback and goal setting. These techniques are aimed at teaching specific skills and promoting parental competence. The intervention was developed on the basis of a review of the scientific literature outlining the key risk and protective 
factors for both parents and infants and includes teaching parenting strategies (Sessions 1 \& 2), individual coping skills (Session 3) and partner support skills (Session 4).

The parenting strategies are designed to promote a warm and responsive relationship between parents and their babies as well as promoting a positive learning environment for the infant. These first four sessions are conducted in a group setting. Specific parenting strategies covered include spending quality time, affection, settling strategies and providing a stimulating and engaging environment. Individual coping skills taught include acceptance, identifying the relationship between thoughts and emotions, using coping statements, abdominal breathing, being involved in pleasant activities, and developing coping plans. As part of the partner support skills session, couples learn positive speaking and listening skills, casual conversations, communicating their emotional experience and spending quality time together. Throughout the group sessions, parents are given information about common experiences of new parents both at an individual as well as on a couple level. This information aims to promote realistic expectations about the changes that come with the transition to parenthood. Parents further learn how to apply parenting strategies appropriately across the different developmental milestones of the infant. During Session 1, parents are provided with a copy of the Baby Triple P workbook which they could take home and review the content in their own time.

The content is followed up with four postnatal individual telephone sessions (Sessions 5 to 8), which employ a self-regulatory model. The telephone sessions review homework from a previous session, focussing on strengths and areas to improve, and setting and monitoring goals for areas of future change. This teaches participants to reflect on their own behavior as well as the impact it has on their baby. Both the prenatal group sessions and the telephone sessions will be delivered by the first author and trained interns in clinical psychology.

\section{Care as usual}

Couples in CAU will complete assessments at baseline, ten weeks post birth, and at six months post birth. These are the same time points at which the intervention group complete their assessments. During this time, families will continue to receive regular primary care and other pre and post-natal services, as appropriate, including antenatal classes, which may be offered at the mother's hospital, as well as other services provided by GPs or obstetricians. These services were also available to mothers in the Baby Triple P group. Other than regarding issues of assessment, these parents will have no contact with the research team, but are free to access support from other community services and will be given appropriate referral information should they ask for assistance. After the 6-month follow-up assessment, families will be offered the Baby Triple P workbook.

\section{Protocol adherence}

Practitioner who deliver the intervention are trained using a standardized system of training and accreditation, designed to promote program use and fidelity. Sessions are delivered according to a standardized manual and practitioners received regular clinical supervision. Each practitioner will complete a protocol adherence checklist for each session conducted, which will be coded by a research assistant who is independent of the delivery of the sessions but familiar with the intervention and protocols for adherence. Using structured session checklists, videotapes of group sessions will be independently coded for protocol adherence. A second rater will code $25 \%$ of the sessions to establish inter-rater reliability (kappa).

\section{Assessment}

Table 1 provides an overview of the assessment measures and the time points at which they are assessed. All parent-report measures will be provided in a written (online or printed) self-administered questionnaire format, and will take approximately 30 to $40 \mathrm{~min}$ to complete. Most of the measures used have been previously validated and are commonly used. Socioeconomic status (e.g., income, occupation status, parent education), age, history of mental illness and ethnic background will be assessed using the Family Background Questionnaire [61]. Only the mother will complete this measure, as well as the Cambridge Worry Scale [CWS; 68].

The CWS will only be completed by the mother because several items focus on the pregnancy and birth, thus this scale would not be appropriate for fathers. Both mother and father will complete all other self-report measures. Most of the validated scales were written for mothers to complete, thus wording will be slightly changed to suit the father's perspective. Where available, internal consistencies from previous studies for mothers and fathers are reported below. Unless otherwise stated, if only one internal consistency is reported, it would refer to mothers' data only. The home observation will only be conducted with the mother and the baby.

\section{Primary outcome measures \\ Parenting confidence and sense of competency}

The Maternal Self-Report Inventory [62] is an overall measure of self-esteem related to the adaptation of motherhood, which has a total of 100 questions across seven subscales. This study will use only the 8-item General Ability and Preparedness for Mothering Role subscale of the 26-item short form of the questionnaire. The 5 point scale ranges from $1=$ completely false to 5 = completely true. This subscale has good internal consistency $(\alpha=.88)$. The full scale has demonstrated acceptable external validity [62]. 
Table 1 Summary of Assessment Measures

\begin{tabular}{|c|c|c|}
\hline Construct & Assessment Measure & Time Point \\
\hline Demographics & Family Background Questionnaire & $\mathrm{T} 1$ \\
\hline \multicolumn{3}{|l|}{ Primary Outcomes } \\
\hline Parental Confidence & Maternal Self-Report Inventory & $\mathrm{T} 1, \mathrm{~T} 2, \mathrm{~T} 3$ \\
\hline Parental Self-Efficacy & Maternal Efficacy Questionnaire & $\mathrm{T} 2, \mathrm{~T} 3$ \\
\hline \multicolumn{3}{|l|}{ Secondary Outcomes } \\
\hline \multicolumn{3}{|l|}{ Parental Mental Health } \\
\hline Depression & Edinburgh Postnatal Depression Inventory & $\mathrm{T} 1, \mathrm{~T} 2, \mathrm{~T} 3$ \\
\hline Anxiety & Depression Anxiety Stress Scale 21 & $\mathrm{~T} 1, \mathrm{~T} 2, \mathrm{~T} 3$ \\
\hline Pregnancy Worry (mothers only) & Cambridge Worry Scale & $\mathrm{T} 1, \mathrm{~T} 2, \mathrm{~T} 3$ \\
\hline Life Satisfaction & Oxford Happiness Questionnaire & $\mathrm{T} 1, \mathrm{~T} 2, \mathrm{~T} 3$ \\
\hline Expectations & Prenatal Maternal Expectations Scale & $\mathrm{T} 1$ \\
\hline \multicolumn{3}{|l|}{ Family and Social Support } \\
\hline Couple Relationship & 3 items from PAFAS, 2 items from HCTC \& 1 overall item & $\mathrm{T} 1, \mathrm{~T} 2, \mathrm{~T} 3$ \\
\hline Social Support & Social Support Scale & $\mathrm{T} 1$ \\
\hline \multicolumn{3}{|l|}{ Mother-Child Relationship } \\
\hline Maternal Responsiveness & Maternal Infant Responsiveness Inventory & $\mathrm{T} 2, \mathrm{~T} 3$ \\
\hline Observed Mother-Infant Responsiveness & The Care Index & $\mathrm{T} 2, \mathrm{~T} 3$ \\
\hline Mother-Child Bonding & Postpartum Bonding Instrument & $\mathrm{T} 2, \mathrm{~T} 3$ \\
\hline \multicolumn{3}{|l|}{ Baby Outcomes } \\
\hline Problematic Behaviors & Baby Behaviour Inventory & $\mathrm{T} 2, \mathrm{~T} 3$ \\
\hline Baby Behaviors & Baby Diary & $\mathrm{T} 2, \mathrm{~T} 3$ \\
\hline \multicolumn{3}{|l|}{ Other } \\
\hline Parent Involvement & Attendance at group sessions (intervention only) & During group sessions \\
\hline Conflicting Advice \& Problem Solving & 6 items & $\mathrm{T} 1, \mathrm{~T} 2, \mathrm{~T} 3$ (problem solving only $\mathrm{T} 2$ and $\mathrm{T} 3$ ) \\
\hline Strategies used & Strategies Checklist & T2 (intervention only), T3 \\
\hline Services accessed & & $\mathrm{T} 2, \mathrm{~T} 3$ \\
\hline Client Satisfaction & Client Satisfaction Scale & $\mathrm{T} 2$ \\
\hline
\end{tabular}

The Maternal SelfEfficacy Scale [MSES; 40] is a 10-item measure capturing parent's selfefficacy and perceived behavioral competence among mothers of infants in the first year. It has previously been used with clinically depressed and nondepressed mothers of infants aged three to 13 months. Nine of the ten items address mother's feelings of competence in relation to specific domains of infant care, for example soothing the baby, understanding what the baby wants, amusing the baby, disengaging from the baby, performing daily routine tasks, such as feeding, changing and bathing the baby. A final tenth item taps mother's global feelings of efficacy in mothering. Responses are recorded on a 4-point scale from $1=n o t$ good at all to $4=v e r y$ good. This subscale has good reliability $[\alpha=.86 ; 40, \alpha=.75$ and .84 , respectively for fathers and mothers; 58] and demonstrated good concurrent validity to the Parenting Sense of Competence Scale [40].

\section{Secondary outcomes}

\section{Parental well-being}

The Edinburgh Postnatal Depression Scale [EPDS; 63] is a 10-item scale and is widely used to screen for parental depression during pregnancy and post-birth. Expectant mothers and fathers are asked to indicate how often they have experienced symptoms relating to depression and/ or anxiety over the previous seven days. The response scale is from $0=n o$, not at all to $3=y e s$, all the time. The EPDS has good internal consistency for mothers $[\alpha=.87$; 63] and fathers $[\alpha=.81]$; [63]. The scale's sensitivity in detecting depression has been shown to be between 80 and $100 \%[64,65]$.

The Depression, Anxiety and Stress Scale 21 [66] is a 21-item measure assessing symptoms of depression, anxiety and stress in adults. This study will only use the anxiety and stress subscales. The 4 point response scale is from 0 $=$ did not apply to me at all, never, to $3=$ applied to me very much, or most of the time, almost always. The measure has 
good reliability for the anxiety $(\alpha=.81)$ and stress $(\alpha=.88)$ subscales for both men and women together. The scale demonstrated good convergent validity with the Mood and Anxiety Symptom Questionnaire 90, the Perceived Stress Scale and the Beck Anxiety Inventory [67].

The Cambridge Worry Scale [68] is a 17-item prenatal measure assessing worries and concerns in relation to pregnancy. Items assess concerns about health, worry about labor and birth, worry about the baby and concerns about relationships. The 6 point response scale is from $0=$ not $a$ worry to $5=$ major worry. The scale has satisfactory internal consistency $(\alpha=.79)$, and good convergent validity with the trait form of the State-Trait Anxiety Inventory [68].

The Oxford Happiness Questionnaire (OHQ; Hills \& Argyle, 2002) is an 8-item measure assessing personal happiness, based on the original Oxford Happiness Inventory [69]. The 6 point Likert Scale is from $1=$ strongly disagree to $6=$ strongly agree. The scale has shown high internal consistency $(\alpha=.91)$ for both men and women, and good convergent validity with variety of measures related to happiness and satisfaction with life [70].

The Prenatal Maternal Expectations Scale [71] has 46 items and assesses prenatal expectations pertaining to the following subdomains: characteristics of the baby and child care; degree of enjoyment anticipated in association with parenting, expected changes in the parent's significant relationships resulting from becoming a parent, anticipated changes in lifestyle or quality of life associated with the parental role, and projected image of self as a parent. Responses are made on a 5 point scale from $1=$ strongly agree to $5=$ strongly disagree. The scale has good reliability $(\alpha=.83)$ and predictive validity [71].

\section{Family and social support}

The Social Support Scale [72] will be used to assess participants' satisfaction with their social network. Two out of the four items will be used for this study. The first item asks participants how satisfied they are with the extent of formal support (i.e., health professionals) they currently receive. The second item referred asks participants how satisfied they are with the extent of informal support (i.e., provided by family, friends and neighbors) they currently receive (from $1=$ Not at all satisfied to 5 = Completely satisfied).

The Couple Relationship will be assessed using three items from the Parenting and Family Adjustment Scale [PAFAS]; [73], two items from the Household and Childcare Task Checklist [74] measuring overall perceived fairness and satisfaction with the division of tasks, and one overall item on global relationship satisfaction from the Relationship Quality Index [75]. For the purpose of the current study, the PAFAS items assessing the couple relationship were slightly reworded to suit the context of expecting/ having a baby.
For both the social support and couple relationship measures, only a selected few items were chosen to be included in order to limit the total length of the questionnaires. The social support measure and one item from the couple relationship measure were intended as a risk factor indicator only to be used for edibility assessment, rather than an outcome measure.

\section{Mother-child relationship}

The Maternal Infant Responsiveness Instrument [76] is a 22item scale designed to measure the parents' feelings about the infant and an appraisal of the infant's responses. Specifically, it measures the parent's recognition of his/her own responses, the parent's recognition of the infant's responses to him/her, and any difficulties they notice in responsiveness. Response options are from $1=$ strongly agree to 5 strongly disagree. Internal consistency of the measure is good $[\alpha=.81$ and .88 for mothers and fathers respectively; $58, \alpha=.86$ for mothers; 75].

The Postpartum Bonding Instrument [77] will assess parentinfant bonding difficulties. This 25-item scale asks parents to rate how often a list of positive and negative thoughts/ emotions are true for them, from 1 =always to $6=$ never. The PBI has four subscales, which reflect impaired bonding, rejection and anger, anxiety about care and risk of abuse. Internal consistency is acceptable $(\alpha=.76)$ and the measure demonstrated good convergent and concurrent validity [78].

The Care Index A home observation will be carried out with the mother and the baby at two stages; upon completion of the program and six months post birth. This will not be done with fathers, as scheduling these visits with fathers will not be practical given that most will be in full-time employment. It will be carried out within the context of semistructured play and naturalistic interaction, lasting approximately $15 \mathrm{~min}$. Mothers will be instructed to interact with their baby as they usually would. With the baby beside her, the mother will first be asked to read an information sheet and the Baby Triple $\mathrm{P}$ brochure for $2 \mathrm{~min}$. This is followed by a nappy change lasting $3 \mathrm{~min}$. The mother will then move on to free play with the baby, using age appropriate toys brought by the researcher. After $5 \mathrm{~min}$, the researcher will prompt the mother to leave the room briefly for a maximum of $2 \mathrm{~min}$. This can be reduced to $20-30 \mathrm{~s}$ if the baby is upset and is not able to settle on his or her own. Lastly, the reunion context will be recorded for 2 min. Observations will be videotaped. Video recording of the mother's interaction with the baby (i.e., the $5 \mathrm{~min}$ component) will be coded using the Care Index [79]. The Care Index assesses mother-infant interaction from birth to about two years of age based on a short, videotaped play interaction of 3-5 $\mathrm{min}$ (from the five minute 
play time segment). Trained independent coders will conduct this assessment, who will be blind to the study aims. The CARE-Index is a dyadic procedure that assesses adult sensitivity in a dyadic context. The measure assesses mothers on three scales: sensitivity, control and unresponsiveness. There are also four scales for infants: cooperativeness, compulsivity, difficultness, and passivity. Validity has been demonstrated with the Parent-infant Interaction Observation Scale [80]. Reliabilities will be assessed by calculating the inter-rater reliabilities for a random $25 \%$ of the coding between the two coders.

\section{Baby outcomes}

The Baby Behaviour Inventory [BBI; 80] measures the extent of a range of behaviors that parents often find challenging during the first twelve months. In addition, it captures the level of parental confidence in dealing with the behavior. Encompassing 14 items, parents indicate how often certain behaviors occur. Parents are then asked to identify whether they have experienced the behavior as a problem with their baby using a yes/no format. If the behavior is rated as a problem, parents are asked to rate their confidence in dealing with the behavior. The overall intensity scale of the BBI was found to have satisfactory internal consistency $(\alpha=.76)$ and has demonstrated construct validity to the EPDS and the MSES [81].

The Baby Diary [82] was modelled on other similar diaries used in previous studies. We will ask parents to fill in a diary for a 24 hour period to identify the pattern of feeding, sleeping, crying and contentedness in babies. Parents will mark the dominant behavior using specific symbols for each of the four behaviors for 48 half-hour time periods from 6 am to 6 am the following day. Only one diary will be completed per time point so that either mother or father can complete it or they can complete it together.

\section{Additional variables}

Parent Attendance will be assessed by the group facilitator taking attendance of both mothers and fathers at the group sessions. This will only be assessed for parents in the intervention group. It will be coded as either present or not present for both mothers and fathers.

The Conflicting Advice Questionnaire and Problem Solving is a 6item measure that was developed for this study to assess the extent participants received conflicting information or advice from professionals and from family and friends, as well as how they deal with confusion and problems. First, it asks about the extent of confusion in regards to five areas, separately from professionals, and from friends and family: feeding the baby, settling the baby, putting the baby to sleep, bathing the baby and dressing and changing the baby. Then, participants are asked to write about any other topics where they experience confusion. Next, participants are asked about what they do when they are confused or encounter a problem in regards to their baby. They can tick one or more of the following options: seek out additional information from family, seek out additional information from professionals, research the internet, corroborate with partner about the problem and try to think of a solution together, try out different strategies until one works. The final item allows participants to write about any other problem solving strategies they have been using.

Strategies checklist This checklist asks parents to indicate which of the 29 strategies that are taught in the Baby Triple P program they have used since their baby was born. The response options include not at all, a little bit, sometimes, a lot and all the time. This measure was specifically created for this study.

Services accessed Parents will be asked which services they have accessed since they had their baby. Ten options will be listed and participants are asked to tick the service they used, how many times they used it, what they used the service for and how helpful they found it (very, somewhat, not helpful).

The Client Satisfaction Questionnaire $[55,83]$ is an 8item questionnaire that assesses participant's perception of the quality of the program received, how well the program addresses their needs as well as their overall satisfaction with the services provided. Only the Baby Triple $\mathrm{P}$ group completed this measure.

\section{Statistical analyses}

Prior to conducting the main analyses, data will be screened for distributional assumptions (e.g., univariate and multivariate normality, outliers and multi-collinearity) as well as inter- and intra-measure consistency. Preliminary analyses will also investigate whether the study groups differ on any demographic characteristics at baseline. This will be examined via linear (for continuous outcomes) and logistic (for categorical outcomes) regression models. If baseline group differences are detected, we will investigate and report the extent to which the results from the planned analyses described below are altered, when these differences are statically controlled. For missing data points, an analysis of missing data will be conducted (see paragraph below on missing data) and imputation methods will be considered.

\section{Primary analyses}

The analyses will evaluate the effects of the intervention on parent and baby outcomes, including the primary outcome measures of parenting confidence and sense of competency. These analyses will include self-reports obtained from parents as well as reports on baby behaviors, baby diaries and observational assessment. A multilevel modeling approach will be used to take into account the 
repeated measurements and thus non-independence of observations [84]. This approach will be used for variables that have three measurement time points (e.g. Couple Relationship and the EPDS). Dummy codes contrasting the groups will serve as fixed effects, allowing for random intercepts and slopes to vary across individuals. Outcome variables with two time points (e.g., MSES, and the Baby Diary) will be analyzed using a series of rANOVAs and rMANOVAs. The primary analyses will be conducted for mother and father data separately to investigate whether the intervention is beneficial for both parents, or has different effects for mothers and fathers. Significant fixed effects contrasting Baby Triple P with CAU will indicate whether the intervention is an improvement over usual care. Separate models will be estimated for each outcome measure using Bonferroni correction to control for inflation of Type 1 error due to multiple comparisons.

\section{Sample size and power analysis}

The required sample size for the study was calculated to assure $80 \%$ power to detect a medium effect size $(E S)=0.5$ for a mean difference in the variables of interest between the groups (INT vs CAU). Effect sizes for early intervention studies range from small to large on a range of outcomes [48]. Two studies that assessed parental confidence and competence as an outcome of a parenting intervention found a small [85] and a large [43] effect. Given these are our primary outcome measures, a medium effect size was expected in this study. Furthermore, as our study will recruit an at-risk sample, an expected medium effect is a conservative estimate. We performed power analysis using G*Power software [86] for rANOVA, looking at the within-between interactions and allowing for conservative estimate of intra-individual variability (.5). The analyses indicated that a sample size of 80 is sufficient to detect an ES of 0.5 at the significance level of .05 (two-tailed). This is without taking into account the added power accorded by the rich repeated measures in our data. In multilevel models with repeated measures, the sample size is effectively the number of observations (level-1), not number of participants (level-2 units). Assuming a 20\% attrition rate (based on estimate within Bakermans-Kranenburg, van Ijzendoorn [46] review), an available sample of 110 families will be sufficient to detect medium sized effects.

\section{Missing outcome data}

The full information maximum likelihood analysis (FIML) approach will be implemented to accommodate for missing data when using a multilevel modeling approach. For other variables analyzed using rANOVA and rMANOA, multiple imputations will be used. If the assumptions of either Missing Completely at Random (MCAR) or Missing at Random (MAR) are met [87], then these approaches will yield estimates consistent with what would be expected if there were no missing data. Thus, it will allow intention-to-treat analysis (inclusion of all randomized families in the analyses).

\section{Discussion}

This protocol paper outlines the background and design of a randomized controlled trial of Baby Triple $P$ for first-time pregnant parents in high-risk populations. It will fill the much-needed gap in the literature as to whether a program that combines parenting, parental wellbeing and the couple relationship is effective in helping high-risk parents ease into the transition to parenthood. This project will employ a rigorous methodology, with multi-domain and multi-informant assessment in order to inform future intervention development and tailoring. Furthermore, by applying an advanced statistical approach we will assist in expanding the knowledge of a variety of intervention outcomes as well as identify aspects for tailoring early parenting interventions in the future. A particular strength of this study will be the recruitment of a high-risk sample that is most likely to benefit from a program, such as Baby Triple P. Moreover, prior intervention studies have largely involved only mothers. This study will encourage both mothers and fathers to attend the intervention together. This will have the added benefit for families, as it will allow for parents to start their parenting journey as a team and research shows that when both parents attend an intervention, the effects are stronger [88, 89]. Further, by evaluating effects for mothers and fathers we will learn whether they benefit from the program differently.

A few limitations of this study should be kept in mind. First, the majority of the assessments are self-report questionnaires, which rely on participant's honesty, accurate self-reflection and memory of events, as well as their understanding and interpretation of the questions. They also are affected by social biases. However, the inclusion of the observational assessment of responsiveness using the Care Index aims to combat some of the biases of the self-report measures. Another limitation could be the use of a care as usual control group in place of an attention-placebo comparison, as any possible changes after the intervention might be due to the additional support and attention received from the facilitator rather than the specific content of Baby Triple P. However, we have included a strategy checklist to identify if and which of the strategies parents are using, and we can therefore ascertain differences between the groups due to the extent the Baby Triple P strategies are utilized. This would be a good indicator that the intervention itself leads to changes in the outcomes. Furthermore, the use of an attention-placebo control group has it's own limitations in preventative parenting intervention research, such as unequal drop outs in the control 
group arm and ethical dilemmas when unsafe parenting behaviors are discussed among parents [90].

Recruitment and enrolment to the study commenced in January 2014 and closed in March 2015. Follow-up data collection was completed in January 2016. During the design phase of this study, the other trials on Baby Triple P discussed earlier were ongoing and results have only become available during the course of this trial. Results will be published in a peer-reviewed journal when analyses are completed. It is expected that participating in a group-based preventative parenting intervention has the potential to help new parents adjust to the transition to parenthood more easily by improving parenting confidence, competence, the parent-infant relationship, parent wellbeing as well as reducing baby behavior problems.

\section{Abbreviations \\ BBI: Baby Behaviour Inventory; CAU: Care as usual; CONSORT: Consolidated standards of reporting trials; CWS: Cambridge Worry Scale; EPDS: Edinburgh Postnatal Depression Scale; ES: Effect size; FIML: Full information maximum likelihood analysis; INT: Intervention; MAR: Missing at random; MCAR: Missing completely at random; MSES: Maternal self-efficacy scale; OHQ: Oxford Happiness Questionnaire; PAFAS: Parenting and Family Adjustment Scale; r(M)ANOVA: repeated measures (Multivariate) Analysis of Variance; Triple P: Positive parenting program}

\section{Funding}

This study did not receive any funding.

\section{Availability of data and materials}

The datasets used and/or analyzed during the current study available from the corresponding author on reasonable request.

\begin{abstract}
Authors' contributions
MM, AM and AF are the chief investigators who designed and established this research study. MM drafted the first version of this manuscript. All authors have made substantial contributions to conception and design, and the writing of the manuscript and have critically drafted, reviewed, revised and approved the final version for important intellectual content. MM was responsible for ethics applications and reporting. MM will be responsible for recruitment and data collection. All authors will be involved in preparation of publications on the clinical outcomes of the study. Each author has participated sufficiently in the work to take public responsibility for appropriate portions of the content; and agreed to be accountable for all aspects of the work in ensuring that questions related to the accuracy or integrity of any part of the work are appropriately investigated and resolved.
\end{abstract}

\section{Ethics approval and consent to participate}

Ethical approval has been obtained from the Queensland Children's Health Services Human Research Ethics Committee (HREC/13/QRBW/177) and the University of Queensland Behavioural and Social Sciences Ethical Review Committee (2013000564).

Participants will receive detailed information before signing up for this study and the research coordinator (MM) is available to discuss any concerns with participants. Consent will be obtained electronically via the trial website. Confidentiality. All study-related information will be stored securely at the study site. All participant information will be stored in locked file cabinets in areas with limited access only by the research team. All, reports, data collection, process, and administrative forms will be identified by a coded ID [identification] number only to maintain participant confidentiality. All records that contain names or other personal identifiers, such as locator forms and informed consent forms, will be stored separately from study records identified by code number. All local databases will be secured with password-protected access systems. Forms, lists, logbooks, appointment books, and any other listings that link participant ID numbers to other identifying information will be stored in a separate, locked file in an area with limited access.

\section{Consent for publication}

Not applicable.

\section{Competing interests}

The authors declare they have the following competing interests. The Parenting and Family Support Centre is partly funded by royalties stemming from published resources of the Triple P - Positive Parenting Program, which is developed and owned by The University of Queensland (UQ). Royalties are also distributed to the Faculty of Health and Behavioural Sciences at UQ and contributory authors of published Triple P resources. Triple P International (TPI) Pty Ltd. is a private company licensed by Uniquest Pty Ltd. on behalf of UQ, to publish and disseminate Triple P worldwide. The authors of this report have no share or ownership of TPI. Dr. Alina Morawska receives royalties from TPI. TPI had no involvement in the study design, collection, analysis or interpretation of data, or writing of this report. Dr. Mandy Mihelic was a student at UQ at the time this study was developed and carried out.

\section{Publisher's Note}

Springer Nature remains neutral with regard to jurisdictional claims in published maps and institutional affiliations.

\section{Author details}

${ }^{1}$ Parenting and Family Support Centre, School of Psychology, The University of Queensland Brisbane, St Lucia, QLD 4067, Australia. ${ }^{2}$ Center for Self-Report Science, Center for Social \& Economic Research, University of Southern California, Los Angeles, USA.

Received: 14 December 2016 Accepted: 13 July 2018 Published online: 28 July 2018

\section{References}

1. Feeney $\mathrm{JA}$, et al. Becoming parents: exploring the bonds between mothers, fathers, and their infants. New York: Cambridge University Press; 2001.

2. Glade AC, Bean RA, Vira R. A prime time for marital/relational intervention: a review of the transition to parenthood literature with treatment recommendations. The American Journal of Family Therapy. 2005;33(4):319-36.

3. Murray L, Cooper PJ. Effects of postnatal depression on infant development. Archives in Diseases in Childhood. 1997;77:99-101.

4. Cornish AM, et al. Postnatal depression and infant cognitive and motor development in the second postnatal year: the impact of depression chronicity and infant gender. Infant Behavior and Development. 2005;28(4):407-17.

5. Doss $\mathrm{BD}$, et al. The effect of the transition to parenthood on relationship quality: an 8-year prospective study. J Pers Soc Psychol. 2009;96(3):601-19.

6. Sanders MR. Development, evaluation, and multinational dissemination of the triple P-positive parenting program. Annu Rev Clin Psychol. 2012;8:345-79.

7. Cowan $\mathrm{CP}$, Cowan PA. When Partners become Parents. The big life change for couples. Mahwah, New Jersey: Lawrence Erlbaum Associates, Inc; 2000.

8. Schulz MS, Cowan CP, Cowan PA. Promoting healthy beginnings: a randomized controlled trial of a preventive intervention to preserve marital quality during the transition to parenthood. J Consult Clin Psychol. 2006; 74(1):20-31.

9. Hemmi MH, Wolke D, Schneider S. Associations between problems with crying, sleeping and/or feeding in infancy and long-term behavioural outcomes in childhood: a meta-analysis. Arch Dis Child. 2011;96(7):622-9.

10. Wolke D, Rizzo P, Woods S. Persistent infant crying and hyperactivity problems in middle childhood. Pediatrics. 2002;109(6):1054-60.

11. Russell $\mathrm{C}$. The state of knowledge about prevention/early intervention. Canada: Invest in Kids; 2002.

12. Hirst DC. 'Rebirthing' - Report of the Review of Maternity Services in Queensland, Q. Health, Editor. Queensland; 2005. https://www.health.qld. gov.au/_data/assets/pdf_file/0024/435660/maternityreview.pdf.

13. Gavin NI, et al. Perinatal depression: a systematic review of prevalence and incidence. Obstet Gynecol. 2005;106(5):1071-83.

14. Heron J, et al. The course of anxiety and depression through pregnancy and the postpartum in a community sample. J Affect Disord. 2004;80(1):65-73.

15. Belsky J, Rovine M. Patterns of marital change across the transition to parenthood: pregnancy to three years postpartum. J Marriage Fam. 1990;52:5-19. 
16. Bronte-Tinkew J, et al. Symptoms of major depression in a sample of fathers of infants: sociodemographic correlates and links to father involvement. J Fam Issues. 2007;28(1):61-99.

17. Dunning MJ, Giallo R. Fatigue, parenting stress, self-efficacy and satisfaction in mothers of infants and young children. J Reproductive Infant Psychology. 2012;30(2):145-59.

18. Giallo R, et al. Father mental health during the early parenting period: results of an Australian population based longitudinal study. Soc Psychiatry Psychiatr Epidemiol. 2012;47(12):1907-16.

19. Giallo R, et al. Factors associated with the course of maternal fatigue across the early postpartum period. J Reproductive Infant Psychol. 2015;33(5):528-44.

20. Matthey $\mathrm{S}$, et al. Diagnosing postpartum depression in mothers and fathers: whatever happened to anxiety? J Affect Disord. 2003;74(2):139-47.

21. Hackel LS, Ruble DN. Changes in the marital relationship after the first baby is born: predicting the impact of expectancy disconfirmation. J Pers Soc Psychol. 1992;62(6):944-57.

22. Grych JH, Fincham FD. Marital conflict and Children's adjustment: a cognitive-contextual framework. Psychol Bull. 1990;108(2):267-90.

23. Davies PT, Cummings EM. Marital conflict and child adjustment: an emotional security hypothesis. Psychol Bull. 1994;116(3):387-411.

24. Bradley RH, Corwyn RF, McAdoo HP, Coll CG. The home environments of children in the United States part I: variations by age, ethnicity, and poverty status. Child Dev. 2001;72(6):1844-67.

25. Conger RD, Ge X, Elder GH, Lorenz FO, Simons RL. Economic stress, coercive family process, and developmental problems of adolescents. Child Dev. 1994;65(2):541-61.

26. Dodge KA, Pettit GS, Bates JE. Socialization mediators of the relation between socioeconomic status and child conduct problems. Child Dev. 1994;65:649-65.

27. Nelson JA, O'Brien M. Does an unplanned pregnancy have long-term implications for mother-child relationships? J Fam Issues. 2011;33(4):506-26.

28. Barker ED, et al. The contribution of prenatal and postnatal maternal anxiety and depression to child maladjustment. Depression Anxiety. 2011;28(8):696-702.

29. Cutrona CE. Social support and stress in the transition to parenthood. J Abnorm Psychol. 1984;93(4):378-90.

30. O'Connor M, et al. The relationship between social capital and depression during the transition to adulthood. Aust J Psychol. 2011;63(1):26-35.

31. Denis A, Ponsin M, Callahan S. The relationship between maternal selfesteem, maternal competence, infant temperament and post-partum blues. Journal of Reproductive and Infant Psychology. 2012;30(4):388-97.

32. Davis EP, et al. Prenatal maternal anxiety predict negative behavioural reactivity in infancy. Infancy. 2004;6(3):319-31.

33. Parfitt $Y$, Pike A, Ayers S. Infant developmental outcomes: a family systems perspective. Infant Child Dev. 2014:23:353-73.

34. Carlson MJ, et al. Couples as partners and parents over children's early years. J Marriage Fam. 2011;73(2):317-34.

35. Davies PT, et al. Child emotional security and interparental conflict. Monogr Soc Res Child Dev. 2002;67(3):X-113.

36. Mihelic M, Filus A, Morawska A. Correlates of prenatal parenting expectations in new mothers: is better self- efficacy a potential target for preventing postnatal adjustment difficulties? Prev Sci. 2016;17(8):949-59.

37. Olioff M, Aboud FE. Predicting postpartum dysphoria in primiparous mothers: roles of perceived parenting self-efficacy and self-esteem. J Cogn Psychother. 1991;5(1):3-14.

38. Cutrona CE, Troutman BR. Social support, infant temperament, and parenting self-efficacy: a mediational model of postpartum depression. Child Dev. 1986;57(6):1507-18.

39. Coleman PK, Karraker KH. Self-efficacy and parenting quality: findings and future applications. Dev Rev. 1997;18:47-85.

40. Teti DM, Gelfand DM. Behavioral competence among mothers of infants in the first year: the mediational role of maternal self-efficacy. Child Dev. 1991; 62:918-29.

41. Bohlin G, Hagekull B. "good mothering": maternal attitudes and motherinfant interaction. Infant Mental Health Journal. 1987:8(4):352-63.

42. de Wolff MS, van ljzendoorn MH. Sensitivity and attachment: a meta-analysis on parental antecedents of infant attachment. Child Dev. 1997;68(4):571-91.

43. Wolfson A, Futterman A, Lacks P. Effects of parent training on infant sleeping patterns, parents' stress, and perceived parental competence. J Consult Clin Psychol. 1992;60(1):41-8.

44. Bell SM, Ainsworth SMD. Infant crying and maternal responsiveness. Child Dev. 1972;43(4):1171-90.
45. Pinquart M, Teubert D. Effects of parenting education with expectant and new parents: a meta-analysis. J Fam Psychol. 2010;24(3):316-27.

46. Bakermans-Kranenburg MJ, van ljzendoorn MH, Juffer F. Less is more: metaanalyses of sensitivity and attachment interventions in early childhood. Psychol Bull. 2003;129(2):195-215.

47. Bryanton J, Beck CT. Postnatal parental education for optimizing infant general health and parent-infant relationships (review). Cochrane Libr. 2010;12:1-90.

48. Mihelic M, Morawska A, Filus A. Effects of early parenting interventions on parents and infants: a meta-analytic review. J Child Fam Stud. 2017;26(6):1507-26.

49. Feinberg ME, et al. Effects of family foundations on parents and children: 3.5 years after baseline. J Fam Psychol. 2010;24(5):532-42.

50. Feinberg ME, Kan ML. Establishing family foundations: intervention effects on coparenting, parent/infant well-being, and parent-child relations. J Fam Psychol. 2008:22(2):253-63.

51. Feinberg ME, Kan ML, Goslin MC. Enhancing coparenting, parenting, and child self-regulation: effects of family foundations 1 year after birth. Prev Sci. 2009;10(3):276-85

52. Petch J, et al. A randomized controlled trial of a couple relationship and coparenting program (couple CARE for Parents) for high- and low-risk new parents. J Consult Clin Psychol. 2012;80(4):662-73.

53. Murray $L$, et al. The impact of postnatal depression and associated adversity on early mother-infant interactions and later infant outcome. Child Dev. 1996;67:2512-26.

54. Spry C, Morawska A, Sanders M. Baby Triple P Group Workwook. Brisbane: Triple P International Pty. Ltd.; 2011.

55. Spry C. The Baby Triple P Project: Effects of a parenting intervention to promote a successful transition to parenthood., in School of Psychology: The University of Queensland; 2013.

56. Ferrari $\mathrm{AJ}$, et al. Prem baby triple $\mathrm{P}$ a new parenting intervention for parents of infants born very preterm: acceptability and barriers. Infant Behav Dev. 2011:34(4):602-9.

57. Butler $\mathrm{H}$, et al. The acceptability and feasibility of the baby triple $\mathrm{P}$ positive parenting Programme on a mother and baby unit: Qmethodology with mothers with severe mental illness. Arch Womens Ment Health. 2014;17:455-63.

58. Tsivos $Z \mathrm{~L}$, et al. A pilot randomised controlled trial to evaluate the feasibility and acceptability of the baby triple $\mathrm{P}$ positive parenting Programme in mothers with postnatal depression. Clinical Child Psychology Psychiatry. 2015;20(4):532-54

59. Seah, C.K.F. and A. Morawska, Does Baby Triple P enhance sensitivity and reduce parenting stress? A randomized controlled trial of an early parenting intervention. Manuscript submitted for publication.

60. Gagnon AJ, Sandall J. Individual or group antenatal education for childbirth or parenthood, or both (review). Cochrane Libr. 2011;10:1-64

61. Sanders MR, Morawska A. Family Background Questionnaire. Brisbane: Parenting and family support Centre, The University of Queensland; 2010.

62. Shea E, Tronick EZ. The maternal self-report inventory. A research and clinical instrument for assessing maternal self-esteem. In: Fitzgerald HE, Lester BM, Yogman MW, editors. Theory and research in behavioral pediatrics. New York: Plenum Press; 1988. p. 101-39.

63. Matthey $\mathrm{S}$, et al. Validation of the Edinburgh postnatal depression scale for men, and comparison of item endorsement with their partners. J Affect Disord. 2001;64:175-84.

64. Cox JL, Holden JM, Sagovsky R. Detection of postnatal depression. Development of the 10-item Edinburgh postnatal depression scale. $\mathrm{Br} \mathrm{J}$ Psychiatry. 1987;150(6):782-6.

65. Boyce P, Stubbs J, Todd A. The Edinburgh postnatal depression scale: validation for an Australian sample. Aust N Z J Psychiatry. 1993;27:472-6.

66. Lovibond PF, Lovibond SH. The structure of negative emotional states: comparison of the depression anxiety stress scales (dass) with the beck depression and anxiety inventories. Behav Res Ther. 1995;33(3):335-43.

67. Osman A, et al. The depression anxiety stress Scales-21 (DASS-21): further examination of dimensions, scale reliability, and correlates. J Clin Psychol. 2012;68(12):1322-38

68. Green JM, et al. Factor structure, validity and reliability of the Cambridge worry scale in a pregnant population. J Health Psychol. 2003;8(6):753-64.

69. Argyle M, Martin M, Crossland J. Happiness as a function of personality and social encounters. In: Forgas JP, Innes JM, editors. Recent advances in social psychology: An international perspective. Amsterdam, North Holland: Elsevier Science; 1989. p. 189-203. 
70. Hills P, Argyle M. The Oxford happiness questionnaire: a compact scale for the measurment of psychological well-being. Personal Individ Differ. 2002; 33:1073-82.

71. Coleman P, Nelson ES, Sundre DL. The relationship between prenatal expectations and postnatal attitudes among first-time mothers. J Reproductive Infant Psychol. 1999;17(1):27-39.

72. Spry C, Morawska A, Sanders MR. Social Support Scale. Brisbane: Parenting and family support Centre, University of Queensland; 2007.

73. Sanders MR, Morawska A. Parenting and Family Adjustment Scale. Brisbane: Parenting and family support Centre, The University of Queensland; 2010.

74. Spry C, Morawska A, Sanders MR. Household and Baby Care Task Checklist. Brisbane: Parenting and family support Centre, University of Queensland; 2007.

75. Norton R. Measuring marital quality: a critical look at the dependent variable. J Marriage Fam. 1983;45(1):141-51.

76. Amankwaa L, Pickler R. Measuring maternal responsiveness. ABNF J. 2007; 18(1):4-15.

77. Brockington IF, et al. A screening questionnaire for mother-infant bonding disorders. Archives Women's Mental Health. 2001;3:133-40.

78. Wittkowski A, Wieck A, Mann S. An evaluation of two bonding questionnaires: a comparison of the mother-to-infant bonding scale with the postpartum bonding questionnaire in a sample of primiparous mothers. Archives Women's Mental Health. 2007:10(4):171-5.

79. Crittenden PM. CARE-Index: Coding Manual, 1979-2004. Miami.

80. Svanberg PO, Barlow A, Tigbe W. The parent-infant interaction observation scale: reliability and validity of a screening tool. J Reproductive Infant Psychol. 2013;31(1):5-14

81. Spry C, Morawska A, Sanders MR. Baby Behaviour Inventory. Brisbane: Parenting and family support Centre, University of Queensland; 2007.

82. Spry C, Morawska A, Sanders MR. Baby Diary. Brisbane: Parenting and family support Centre, University of Queensland; 2007.

83. Sanders MR, Markie-Dadds C, Turner KMT. Practitioner's manual for Standard Triple P. Milton: QLD: Families International; 2001

84. Maas CJM, Hox JJ. Sufficient sample sizes for multilevel modeling. Methodo Europ J Res Methods Behavioral Soc Sci. 2005;1(3):86-92.

85. Barlow $\mathrm{A}$, et al. Effect of a paraprofessional home-visiting intervention on american indian teen mothers' and infants' behavioral risks: a randomized controlled trial. Am J Psychiatr. 2013;170:83-93.

86. Faul F, et al. Statistical power analyses using $G^{*}$ power 3.1: tests for correlation and regression analyses. Behav Res Methods. 2009:41:1149-60.

87. Enders CK. Applied missing data analysis. New York: Guildford Press; 2010.

88. Lundahl BW, et al. A meta-analysis of father involvement in parent training. Res Soc Work Pract. 2007;18(2):97-106.

89. Bagner DM, Eyberg SM. Father involvement in parent training: when does it matter? J Clin Child Adolesc Psychol. 2003;32(4):599-605.

90. Popp L, Schneider S. Attention placebo control in randomized controlled trials of psychosocial interventions: theory and practice. Trials. 2015;16:150.

Ready to submit your research? Choose BMC and benefit from:

- fast, convenient online submission

- thorough peer review by experienced researchers in your field

- rapid publication on acceptance

- support for research data, including large and complex data types

- gold Open Access which fosters wider collaboration and increased citations

- maximum visibility for your research: over $100 \mathrm{M}$ website views per year

At BMC, research is always in progress.

Learn more biomedcentral.com/submissions 